C2. Thrombotic workup revealed mutations of the methylenetetrahydrofolate reductase gene (MTHFR). Treatment with aspirin and folic acid and rehabilitation were followed by improvement in ambulation, but no resolution of decreased proprioception and vibration sense in toes and knees.

Risk factors for SCI without vertebral fracture in pediatric cases reviewed in the literature (Ovid MEDLINE search) include, in decreasing order of frequency, minor trauma (18 cases), bacterial meningitis (15), fibrocartilaginous embolism (10), umbilical artery catheters (8), cardiac arrest with hypotension (6), lumbar puncture with cardiac arrest (6), arteriovenous malformation (6), thrombotic disorders (4), with a total of 108 cases. (Nance JR, Golomb MR. Ischemic spinal cord infarction in children without vertebral fracture. Pediatr Neurol April 2007;36:209-216). (Respond: Dr Golomb, Indiana University School of Medicine, Building XE 040, 575 West Dr, Indianapolis, IN 46202).

COMMENT. Spinal cord infarction (SCI) is rare both in children and adults. In adults, the most common etiologies are aortic aneurysm repair, traumatic aortic rupture, arteriovenous malformation, transient ischemic attack, and cardiac arrest. In a recent report of SCI in an adult, multiple etiologic factors were involved, including diabetes mellitus, hypertension, episodic hypotension, and degenerative disease of the spine (Millichap JJ et al. J Gen Intern Med 2007;22:151-154). These authors provide an anatomical classification of spinal vascular syndromes. When applied to the above pediatric patients, case 1 had an anterior spinal artery syndrome, and case 2 had involvement of both anterior and posterior spinal artery territories. The respiratory distress experienced by patient 1 was localized to C3C5 segments and diaphragmatic paralysis. Homocysteinuria and thromboembolism are reported with MTHFR mutations, accounting for one additional case of SCI in an adolescent while weight-lifting. Other unusual presentations of SCI include congenital cervical spinal atrophy with arthrogryposis multiplex, and symmetrical severe muscle weakness and wasting confined to the upper extremities. (Kaiboriboon K, Hayat GR. Neuropediatrics 2001;32:330-334; Ebinger F et al. Neuropediatrics 2003;34:45-51).

\title{
IDIOPATHIC INTRACRANIAL HYPERTENSION
}

Sex distribution and frequency of obesity in children with idiopathic intracranial hypertension were evaluated at Meyer Children Hospital, Rappaport School of Medicine, Haifa, and other centers in Israel. Meta-analysis was conducted in 244 patients reported since $1997 ; 132(54 \%)$ were younger than 11 years of age and 112 were age 12-17 years. Females were $44 \%$ in the younger group and $70 \%$ in the older group. Obesity was present in $26 \%$ of younger and in $64 \%$ of older patients. Differences in age at presentation, sex, and obesity were statistically significant $(\mathrm{P}<0.01)$. Sex and obesity were independent variables. (Genizi J, Lahat $\mathrm{E}$, Zelnik $\mathrm{N}$ et al. Childhood-onset idiopathic intracranial hypertension: relation of sex and obesity. Pediatr Neurol April 2007;36:247-249). (Respond: Eli Shahar MD, Child Neurology Unit \& Epilepsy Service, Meyer Children Hospital, Rambam Medical Center, Haifa 31096, Israel).

COMMENT. These results in a large series of patients confirm previous findings of differences in age, sex, and obesity in children and adolescents with idiopathic intracranial hypertension. Older patients have higher rates of females affected and of obesity. Intracranial 
hypertension in younger patients may have a different mechanism, no female sex preponderance, and no increased frequency of obesity, when compared to adolescent patients.

\section{METABOLIC DISORDERS}

\section{INFANTILE NEUROGLYCOPENIA}

Participant 1, a 23-year-old woman with congenital hypoglycemia (hyperinsulinism); participant 2, a 16-year-old boy with genetic mutation of the cerebral glucose transporter type 1 (GLUT1 deficient); and participant 3, the 23-year-old healthy twin sister of participant 1 as a control, received a neurologic examination, PET scan, and neuropsychological evaluation, in a study at Neurological Institute, New York, NY. Pt 1 with a residual encephalopathy at 23 years showed hypertonicity, ataxia, dysarthria, hyperreflexia with ankle clonus and Babinski signs. Pt 2 had residual epileptic encephalopathy at 16 years, he received special education, and the neurologic examination was identical to that of pt 1. Treatment with diazoxide increased glycemia and resulted in improved alertness and psychomotor coordination. Pt 3 had a normal neurologic exam and PET scan. PET scans of Pts 1 and 2 were identical, showing hypometabolic activity in parietal, temporal, and thalamic regions, and enhancement of uptake in the basal ganglia, results typical of GLUT1 deficiency. Wechsler scale IQ scores were Pt 1, 64; Pt 2, 49; and Pt 3, 104. Pts 1 and 2 had impaired cognitive skills compared to the healthy twin, Pt 3.

The syndrome of neuroglycopenia is characterized by infantile epilepsy, mental retardation, abnormal neurologic exam and PET scan, all caused by a persistent decrease in glucose supply to the developing brain. The glycopenia may be the result of congenital hypoglycemia or GLUT1 deficiency, and both are treatable diseases. Patients with a milder GLUT1 phenotype are responsible for the familial autosomal dominant transmission of the disease. Diagnosis is made by the typical neurologic manifestations and lumbar puncture showing hypoglycorrhachia. (Pascual JM, Wang D, Hinton V et al. Brain glucose supply and the syndrome of infantile neuroglycopenia. Arch Neurol April 2007;64:507-513). (Respond: Juan M Pascual MD, PhD, Department of Neurology, University of Texas Southwestern Medical Center, 5323 Harry Hines Blvd, Mail Code 8813, Dallas, TX 75390).

COMMENT. Neuroglycopenia may be caused by GLUT1 deficiency or infantile hypoglycemia. The neurologic, psychological, and functional imaging characteristics are similar for both disorders. Early hyperexcitability and seizures are associated with encephalopathy and pyramidal and cerebellar dysfunction. Glucose may have a dual function: 1) as a fuel (energy supply), hypoglycemia resulting in acute neurologic deficits, and 2) as a necessary substrate for normal thalamocortical maturation and development of the nervous system. 